\title{
Article \\ On the Stability of a Generalized Fréchet Functional Equation with Respect to Hyperplanes in the Parameter Space
}

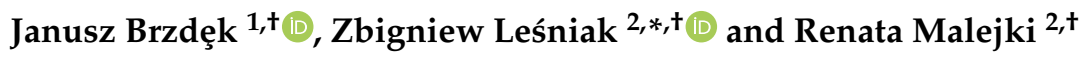 \\ 1 Faculty of Applied Mathematics, AGH University of Science and Technology, Mickiewicza 30, \\ 30-059 Kraków, Poland; brzdek@agh.edu.pl \\ 2 Department of Mathematics, Pedagogical University, Podchorążych 2, 30-084 Kraków, Poland; \\ renata.malejki1@student.up.krakow.pl \\ * Correspondence: zbigniew.lesniak@up.krakow.pl \\ + These authors contributed equally to this work.
}

check for updates

Citation: Brzdęk, J.; Leśniak, Z.; Malejki, R. On the Stability of a Generalized Fréchet Functional Equation with Respect to Hyperplanes in the Parameter Space. Symmetry 2021, 13, 384. https:// doi.org/10.3390/sym13030384

Academic Editor: Sun Young Cho

Received: 8 February 2021

Accepted: 24 February 2021

Published: 27 February 2021

Publisher's Note: MDPI stays neutral with regard to jurisdictional claims in published maps and institutional affiliations.

Copyright: (c) 2021 by the authors. Licensee MDPI, Basel, Switzerland. This article is an open access article distributed under the terms and conditions of the Creative Commons Attribution (CC BY) license (https:/ / creativecommons.org/licenses/by/ $4.0 /)$.

\begin{abstract}
We study the Ulam-type stability of a generalization of the Fréchet functional equation Our aim is to present a method that gives an estimate of the difference between approximate and exact solutions of this equation. The obtained estimate depends on the values of the coefficients of the equation and the form of the control function. In the proofs of the main results, we use a fixed point theorem to get an exact solution of the equation close to a given approximate solution.
\end{abstract}

Keywords: stability; inner product space; fixed point theorem; Fréchet equation

\section{Introduction}

In this paper, we study the functional equation:

$$
A_{1} F(x+y+z)+A_{2} F(x)+A_{3} F(y)+A_{4} F(z)=A_{5} F(x+y)+A_{6} F(x+z)+A_{7} F(y+z),
$$

where $A_{1}, \ldots, A_{7} \in \mathbb{K}$ are constants and $\mathbb{K}$ denotes the fields of real or complex numbers, in the class of functions $F: X \rightarrow Y$ from a commutative group $X$ into a Banach space $Y$ over the field $\mathbb{K}$. This equation is a generalization of the following equation:

$$
F(x+y+z)+F(x)+F(y)+F(z)=F(x+y)+F(x+z)+F(y+z) \text {. }
$$

Equation (2) was used by Fréchet [1] to obtain a characterization the inner product spaces among normed linear spaces, and it is called the Fréchet functional equation. For more results concerning the relationship of Equation (2) with inner product spaces, we refer to [2-8]. Equation (1) is a linear generalization of Equation (2). A nonlinear generalization of the Fréchet functional Equation (2) was considered in [9].

The set of solutions of Equation (1) was studied in [10]. The main result of that paper says that if $A_{i} \neq A_{j}$ for some $i, j \in\{1, \ldots, 7\}$, then each solution of Equation (1) such that $F(0)=0$ is an additive function. In fact, under the assumption that $A_{i} \neq A_{j}$ for some $i, j \in\{1, \ldots, 7\}$, every solution $F$ of Equation (1) is of the form $F=a+c$, where $a$ is an additive function and $c$ is a constant.

In this paper, we investigate the problem of the stability of Equation (1) considering possible values of coefficients $A_{i}$ for $i \in\{1, \ldots, 7\}$. Roughly speaking, for an approximate solution of Equation (1), we are looking for an exact solution of this equation that is close to the given approximate solution. Some results in this direction obtained under assumptions on some coefficients $A_{i}$ can be found in $[10,11]$. The Ulam-type stability problem for functional, difference, differential, and integral equations was described in more detail in the monographs [12-14] and survey papers [15,16]. For a comparison of the stability results for functional equations related to the functional equation considered here, the reader is also referred to [17-28]. 
Equation (1) can be treated as a special case of the general linear equation. The stability problem of the general linear equation was studied in [29-32]. In this article, we want to look at Equation (1) in order to get estimates of the difference between approximate and exact solutions more closely connected to the values of the coefficients of the equation and the form of the control function.

Let us consider the following system of linear equations:

$$
\left\{\begin{array}{l}
A_{2}+A_{3}+A_{4}=0 \\
A_{1}+A_{2}+A_{3}=0 \\
A_{1}+A_{2}+A_{4}=0 \\
A_{1}+A_{3}+A_{4}=0 \\
-A_{2}-A_{3}+A_{6}+A_{7}=0 \\
-A_{2}-A_{4}+A_{5}+A_{7}=0 \\
-A_{3}-A_{4}+A_{5}+A_{6}=0
\end{array}\right.
$$

Its matrix is the form:

$$
\left[\begin{array}{rrrrrrr}
0 & 1 & 1 & 1 & 0 & 0 & 0 \\
1 & 1 & 1 & 0 & 0 & 0 & 0 \\
1 & 1 & 0 & 1 & 0 & 0 & 0 \\
1 & 0 & 1 & 1 & 0 & 0 & 0 \\
0 & -1 & -1 & 0 & 0 & 1 & 1 \\
0 & -1 & 0 & -1 & 1 & 0 & 1 \\
0 & 0 & -1 & -1 & 1 & 1 & 0
\end{array}\right]
$$

The determinant of this matrix is equal to six. Therefore, in the case where not all parameters $A_{1}, \ldots, A_{7}$ are equal to zero, at least one of the equations of System (3) is not satisfied.

In [10], the stability of Equation (1) was proven under the assumption that $A_{2}+A_{3}+$ $A_{4} \neq 0$. In this paper, we consider the remaining six cases corresponding to the equations of System (3). They can be grouped into two classes so that each would contain similar cases. The division into classes is made due to the symmetry of substitutions for the variables occurring in Equation (1). We formulate stability results for one case from each class.

Now, we list the appropriate substitutions, the equations obtained from Equation (1) by using these substitutions, and the form of an operator that can be used in a proof of the stability result corresponding to consecutive cases:

(I) $x=t, y=t, z=t$

$$
\begin{gathered}
A_{1} F(3 t)+\left(A_{2}+A_{3}+A_{4}\right) F(t)=\left(A_{5}+A_{6}+A_{7}\right) F(2 t), \\
F(t)=\frac{A_{5}+A_{6}+A_{7}}{A_{2}+A_{3}+A_{4}} F(2 t)-\frac{A_{1}}{A_{2}+A_{3}+A_{4}} F(3 t)
\end{gathered}
$$

(II)

$$
\begin{aligned}
& x=t, y=t, z=-t \\
& \quad\left(A_{1}+A_{2}+A_{3}\right) F(t)+A_{4} F(-t)=A_{5} F(2 t)+\left(A_{6}+A_{7}\right) F(0),
\end{aligned}
$$

$$
F(t)=\frac{A_{5}}{A_{1}+A_{2}+A_{3}} F(2 t)+\frac{A_{6}+A_{7}}{A_{1}+A_{2}+A_{3}} F(0)-\frac{A_{4}}{A_{1}+A_{2}+A_{3}} F(-t),
$$

$$
\begin{aligned}
& x=t, y=-t, z=t \\
& \left(A_{1}+A_{2}+A_{4}\right) F(t)+A_{3} F(-t)=\left(A_{5}+A_{7}\right) F(0)+A_{6} F(2 t), \\
F(t)= & \frac{A_{5}+A_{7}}{A_{1}+A_{2}+A_{4}} F(0)+\frac{A_{6}}{A_{1}+A_{2}+A_{4}} F(2 t)-\frac{A_{3}}{A_{1}+A_{2}+A_{4}} F(-t),
\end{aligned}
$$

$$
\begin{aligned}
& x=-t, y=t, z=t \\
& \left(A_{1}+A_{3}+A_{4}\right) F(t)+A_{2} F(-t)=\left(A_{5}+A_{6}\right) F(0)+A_{7} F(2 t),
\end{aligned}
$$




$$
F(t)=\frac{A_{5}+A_{6}}{A_{1}+A_{3}+A_{4}} F(0)+\frac{A_{7}}{A_{1}+A_{3}+A_{4}} F(2 t)-\frac{A_{2}}{A_{1}+A_{3}+A_{4}} F(-t),
$$

$$
x=t, y=t, z=0
$$

$$
\left(A_{6}+A_{7}-A_{2}-A_{3}\right) F(t)=\left(A_{1}-A_{5}\right) F(2 t)+A_{4} F(0),
$$

$$
F(t)=\frac{A_{1}-A_{5}}{A_{6}+A_{7}-A_{2}-A_{3}} F(2 t)+\frac{A_{4}}{A_{6}+A_{7}-A_{2}-A_{3}} F(0),
$$

$$
x=t, y=0, z=t
$$

$$
\left(A_{5}+A_{7}-A_{2}-A_{4}\right) F(t)=\left(A_{1}-A_{6}\right) F(2 t)+A_{3} F(0),
$$

$$
F(t)=\frac{A_{1}-A_{6}}{A_{5}+A_{7}-A_{2}-A_{4}} F(2 t)+\frac{A_{3}}{A_{5}+A_{7}-A_{2}-A_{4}} F(0),
$$

$$
\begin{aligned}
& x=0, y=t, z=t \\
& \left(A_{5}+A_{6}-A_{3}-A_{4}\right) F(t)=\left(A_{1}-A_{7}\right) F(2 t)+A_{2} F(0), \\
& F(t)=\frac{A_{1}-A_{7}}{A_{5}+A_{6}-A_{3}-A_{4}} F(2 t)+\frac{A_{2}}{A_{5}+A_{6}-A_{3}-A_{4}} F(0) .
\end{aligned}
$$

As mentioned above, Case (I) was considered in [10]. In this paper, we deal with Cases (II) and (V) chosen from classes consisting of Cases (II)-(IV) and (V)-(VII), respectively. The remaining cases in each class are analogous to those selected.

The proofs of our results are based on the fixed point theorem quoted below. The fixed point approach to the Ulam-type stability problem can also be found in, e.g., [33-36].

Theorem 1 ([37]). Let the following three hypotheses be valid.

(H1) $S$ is a nonempty set; $E$ is a Banach space; and functions $f_{1}, \ldots, f_{k}: S \rightarrow S$ and $l_{1}, \ldots, l_{k}: S \rightarrow$ $\mathbb{R}_{+}$are given, where $\mathbb{R}_{+}$denotes the set of nonnegative reals.

(H2) $\mathcal{T}: E^{S} \rightarrow E^{S}$ is an operator satisfying the inequality:

$$
\|\mathcal{T} \xi(x)-\mathcal{T} \mu(x)\| \leq \sum_{i=1}^{k} l_{i}(x)\left\|\xi\left(f_{i}(x)\right)-\mu\left(f_{i}(x)\right)\right\|, \quad \xi, \mu \in E^{S}, x \in S .
$$

(H3) $\Lambda: \mathbb{R}_{+}{ }^{S} \rightarrow \mathbb{R}_{+}{ }^{S}$ is defined by:

$$
\Lambda \delta(x):=\sum_{i=1}^{k} l_{i}(x) \delta\left(f_{i}(x)\right), \quad \delta \in \mathbb{R}_{+}{ }^{S}, x \in S .
$$

Assume that functions $\varepsilon: S \rightarrow \mathbb{R}_{+}$and $\varphi: S \rightarrow E$ fulfil the following two conditions:

$$
\begin{aligned}
& \|\mathcal{T} \varphi(x)-\varphi(x)\| \leq \varepsilon(x), \quad x \in S, \\
& \varepsilon^{*}(x):=\sum_{n=0}^{\infty} \Lambda^{n} \varepsilon(x)<\infty, \quad x \in S .
\end{aligned}
$$

Then, there exists a unique fixed point $\psi$ of $\mathcal{T}$ with:

$$
\|\varphi(x)-\psi(x)\| \leq \varepsilon^{*}(x), \quad x \in S .
$$

Moreover,

$$
\psi(x):=\lim _{n \rightarrow \infty} \mathcal{T}^{n} \varphi(x), \quad x \in S
$$




\section{The Main Results}

In this section, we prove the stability results for two chosen cases from the above list. In Case (II), we assume that $X$ is a commutative group. However, in Case (V), we can work under the more general assumption. Namely, similar to Case (I) considered in [10], we assume that $X$ is a commutative monoid.

We start with Case (II), which corresponds to the second equation of System (3).

Theorem 2. Let $(X,+)$ be an abelian group, $Y$ be a Banach space, and $A_{1}, \ldots, A_{7} \in \mathbb{K} \in\{\mathbb{R}, \mathbb{C}\}$. Assume that $A_{1}+A_{2}+A_{3} \neq 0$. Let a function $L: X^{3} \rightarrow[0, \infty)$ satisfy the condition:

$$
L(k x, k y, k z) \leq c(k) L(x, y, z), \quad(x, y, z) \in X^{3}, k \in\{2,0,-1\},
$$

with $c(2), c(0), c(-1) \in[0, \infty)$ such that $b:=c(2) d(2)+c(0) d(0)+c(-1) d(-1)<1$, where:

$$
d(2):=\left|\frac{A_{5}}{A_{1}+A_{2}+A_{3}}\right|, d(0):=\left|\frac{A_{6}+A_{7}}{A_{1}+A_{2}+A_{3}}\right|, d(-1):=\left|\frac{A_{4}}{A_{1}+A_{2}+A_{3}}\right| .
$$

Assume that $f: X \rightarrow Y$ is a function such that:

$$
\begin{gathered}
\| A_{1} f(x+y+z)+A_{2} f(x)+A_{3} f(y)+A_{4} f(z)-A_{5} f(x+y)-A_{6} f(x+z) \\
-A_{7} f(y+z) \| \leq L(x, y, z), \quad(x, y, z) \in X^{3} .
\end{gathered}
$$

Then, there exists a unique function $F: X \rightarrow Y$ satisfying (1) such that:

$$
\|f(x)-F(x)\| \leq \rho_{L}(x), \quad x \in X,
$$

where:

$$
\rho_{L}(x):=\frac{L(x, x,-x)}{\left|A_{1}+A_{2}+A_{3}\right|(1-b)}, \quad x \in X .
$$

Proof. Taking $x=y=t, z=-t$ in (18), we obtain:

$$
\left\|\left(A_{1}+A_{2}+A_{3}\right) f(t)+A_{4} f(-t)-A_{5} f(2 t)-\left(A_{6}+A_{7}\right) f(0)\right\| \leq L(t, t,-t), \quad t \in X .
$$

Hence, for each $x \in X$ :

$$
\left\|f(t)+\frac{A_{4}}{A_{1}+A_{2}+A_{3}} f(-t)-\frac{A_{5}}{A_{1}+A_{2}+A_{3}} f(2 t)-\frac{A_{6}+A_{7}}{A_{1}+A_{2}+A_{3}} f(0)\right\| \leq \varepsilon(t),
$$

where $\varepsilon(t):=\frac{L(t, t,-t)}{\left|A_{1}+A_{2}+A_{3}\right|}$. Put:

$$
\mathcal{T} \xi(t):=\frac{A_{5}}{A_{1}+A_{2}+A_{3}} \xi(2 t)+\frac{A_{6}+A_{7}}{A_{1}+A_{2}+A_{3}} \xi(0)-\frac{A_{4}}{A_{1}+A_{2}+A_{3}} \xi(-t), \quad \xi \in Y^{X}, t \in X
$$

Let us note that the operator $\mathcal{T}$ is linear. From (21), we get that:

$$
\|f(t)-\mathcal{T} f(t)\| \leq \varepsilon(t), \quad t \in X
$$


Fix $\xi, \mu \in Y^{X}$. For every $t \in X$, we have:

$$
\begin{aligned}
\|\mathcal{T} \xi(t)-\mathcal{T} \mu(t)\|= & \| \frac{A_{5}}{A_{1}+A_{2}+A_{3}}(\xi(2 t)-\mu(2 t))+\frac{A_{6}+A_{7}}{A_{1}+A_{2}+A_{3}}(\xi(0)-\mu(0)) \\
& -\frac{A_{4}}{A_{1}+A_{2}+A_{3}}(\xi(-t)-\mu(-t)) \| \\
\leq & \left|\frac{A_{5}}{A_{1}+A_{2}+A_{3}}\right|\|\xi(-2 t)-\mu(-2 t)\|+\left|\frac{A_{6}+A_{7}}{A_{1}+A_{2}+A_{3}}\right|\|\xi(0)-\mu(0)\| \\
& +\left|\frac{A_{4}}{A_{1}+A_{2}+A_{3}}\right|\|\xi(-t)-\mu(-t)\| .
\end{aligned}
$$

Thus:

$$
\begin{aligned}
\| \mathcal{T} \xi(t) & -\mathcal{T} \mu(t)\|\leq d(2)\| \xi(2 t)-\mu(2 t)\|+d(0)\| \xi(0)-\mu(0) \| \\
& +d(-1)\|\xi(-t)-\mu(-t)\|, \quad t \in X
\end{aligned}
$$

We showed that Condition (H2) is satisfied with $k=3, S=X, E=Y$,

$$
\begin{aligned}
& f_{1}(t)=2 t, \quad f_{2}(t)=0, \quad f_{3}(t)=-t ｝ \\
{l_{1}(t)=d(2), \quad l_{2}(t)=d(0), \quad l_{3}(t)=d(-1),}
\end{aligned}
$$

i.e.,:

$$
\|\mathcal{T} \xi(t)-\mathcal{T} \mu(t)\| \leq \sum_{i=1}^{3} l_{i}(t)\left\|\xi\left(f_{i}(t)\right)-\mu\left(f_{i}(t)\right)\right\|, \quad \xi, \eta \in Y^{X}, t \in X
$$

Define an operator $\Lambda: \mathbb{R}_{+}{ }^{X} \rightarrow \mathbb{R}_{+}{ }^{X}$ by:

$$
\Lambda \eta(t):=\sum_{i=1}^{3} l_{i}(t) \eta\left(f_{i}(t)\right), \quad t \in X
$$

for every $\eta \in \mathbb{R}_{+}{ }^{X}$. Then, for each $\eta \in \mathbb{R}_{+}{ }^{X}$, we have:

$$
\Lambda \eta(t)=d(2) \eta(2 t)+d(0) \eta(0)+d(-1) \eta(-t), \quad t \in X .
$$

Let us note that the operator $\Lambda$ is monotone, i.e., for all $\eta, \zeta \in \mathbb{R}_{+}{ }^{X}$, if $\eta \leq \zeta$, then $\Lambda \eta \leq \Lambda \zeta$. Moreover, by (23):

$$
\|\mathcal{T} \xi(t)-\mathcal{T} \mu(t)\| \leq \Lambda(\|\xi-\mu\|)(t), \quad \xi, \mu \in Y^{X}, t \in X .
$$


Now, we show that $\varepsilon^{*}(t):=\sum_{n=0}^{\infty} \Lambda^{n} \varepsilon(t)<\infty$ for each $t \in X$, i.e., the function series $\sum_{n=0}^{\infty} \Lambda^{n} \varepsilon(t)$ is convergent for each $t \in X$. Fix a $t \in X$. In view of (16), we have:

$$
\begin{aligned}
\Lambda \varepsilon(t)= & d(2) \varepsilon(2 t)+d(0) \varepsilon(0)+d(-1) \varepsilon(-t) \\
= & d(2) \frac{L(2 t, 2 t,-2 t))}{\left|A_{1}+A_{2}+A_{3}\right|}+d(0) \frac{L(0,0,0)}{\left|A_{1}+A_{2}+A_{3}\right|} \\
& +d(-1) \frac{L(-(t, t,-t))}{\left|A_{1}+A_{2}+A_{3}\right|} \\
\leq & d(2) c(2) \frac{L(t, t,-t)}{\left|A_{1}+A_{2}+A_{3}\right|}+d(0) c(0) \frac{L(t, t,-t)}{\left|A_{1}+A_{2}+A_{3}\right|} \\
& +d(-1) c(-1) \frac{L(t, t,-t)}{\left|A_{1}+A_{2}+A_{3}\right|} \\
= & (d(2) c(2)+d(0) c(0)+d(-1) c(-1)) \frac{L(t, t,-t)}{\left|A_{1}+A_{2}+A_{3}\right|} .
\end{aligned}
$$

Thus:

$$
\Lambda \varepsilon(t) \leq b \varepsilon(t), \quad t \in X
$$

By induction, we show that:

$$
\Lambda^{n} \varepsilon(t) \leq b^{n} \varepsilon(t), \quad t \in X, n \in \mathbb{N} .
$$

For $n=1$, Condition (26) coincides with Condition (25). For $n=2$, by the monotonicity and linearity of $\Lambda$, we get from (25):

$$
\Lambda^{2} \varepsilon(t)=\Lambda(\Lambda \varepsilon)(t) \leq \Lambda(b \varepsilon)(t)=b \Lambda \varepsilon(t) \leq b^{2} \varepsilon(t)
$$

for all $t \in X$. Now, suppose that (26) holds for some $n \in \mathbb{N}$. Then, for every $t \in X$, we have:

$$
\Lambda^{n+1} \varepsilon(t)=\Lambda\left(\Lambda^{n} \varepsilon\right)(t) \leq \Lambda\left(b^{n} \varepsilon\right)(t)=b^{n} \Lambda \varepsilon(t) \leq b^{n+1} \varepsilon(t)
$$

From (26), we receive the following estimate for each $t \in X$ :

$$
\varepsilon^{*}(t)=\sum_{n=0}^{\infty} \Lambda^{n} \varepsilon(t) \leq \sum_{n=0}^{\infty} b^{n} \varepsilon(t)=\frac{\varepsilon(t)}{1-b}=\frac{L(t, t,-t)}{\left|A_{1}+A_{2}+A_{3}\right|(1-b)} .
$$

By Theorem 1 (with $S=X$ and $E=Y$ ), there exists a function $F: X \rightarrow Y$ such that:

$$
F(t)=\frac{A_{5}}{A_{1}+A_{2}+A_{3}} F(2 t)+\frac{A_{6}+A_{7}}{A_{1}+A_{2}+A_{3}} F(0)-\frac{A_{4}}{A_{1}+A_{2}+A_{3}} F(-t), \quad t \in X,
$$

and:

$$
\|f(t)-F(t)\| \leq \varepsilon^{*}(t) \leq \frac{L(t, t,-t)}{\left|A_{1}+A_{2}+A_{3}\right|(1-b)}, \quad t \in X
$$

Moreover,

$$
F(t)=\lim _{n \rightarrow \infty} \mathcal{T}^{n} f(t), \quad t \in X
$$

Next, by induction, we show that for every $(x, y, z) \in X^{3}, n \in \mathbb{N}_{0}:=\mathbb{N} \cup\{0\}$ :

$$
\begin{aligned}
\| A_{1} \mathcal{T}^{n} f(x+y+z) & +A_{2} \mathcal{T}^{n} f(x)+A_{3} \mathcal{T}^{n} f(y)+A_{4} \mathcal{T}^{n} f(z) \\
& -A_{5} \mathcal{T}^{n} f(x+y)-A_{6} \mathcal{T}^{n} f(x+z)-A_{7} \mathcal{T}^{n} f(y+z) \| \\
\leq & b^{n} L(x, y, z), \quad(x, y, z) \in X^{3} .
\end{aligned}
$$


For $n=0$, Condition (29) is simply (18). For $n=1$ using (22), we have:

$$
\begin{aligned}
& \| A_{1} \mathcal{T} f(x+y+z)+A_{2} \mathcal{T} f(x)+A_{3} \mathcal{T} f(y)+A_{4} \mathcal{T} f(z) \\
& A_{5} \mathcal{T} f(x+y)-A_{6} \mathcal{T} f(x+z)-A_{7} \mathcal{T} f(y+z) \| \\
& \left.=\| \frac{A_{5}}{A_{1}+A_{2}+A_{3}} A_{1} f(2(x+y+z))+\frac{A_{6}+A_{7}}{A_{1}+A_{2}+A_{3}} A_{1} f(0)\right) \\
& -\frac{A_{4}}{A_{1}+A_{2}+A_{3}} A_{1} f(-(x+y+z)) \\
& +\frac{A_{5}}{A_{1}+A_{2}+A_{3}} A_{2} f(2 x)+\frac{A_{6}+A_{7}}{A_{1}+A_{2}+A_{3}} A_{2} f(0) \\
& -\frac{A_{3}}{A_{1}+A_{2}+A_{3}} A_{2} f(-x) \\
& +\frac{A_{5}}{A_{1}+A_{2}+A_{3}} A_{3} f(2 y)+\frac{A_{6}+A_{7}}{A_{1}+A_{2}+A_{3}} A_{3} f(0) \\
& -\frac{A_{4}}{A_{1}+A_{2}+A_{3}} A_{3} f(-y) \\
& +\frac{A_{5}}{A_{1}+A_{2}+A_{3}} A_{4} f(2 z)+\frac{A_{6}+A_{7}}{A_{1}+A_{2}+A_{3}} A_{4} f(0) \\
& -\frac{A_{4}}{A_{1}+A_{2}+A_{3}} A_{4} f(-z) \\
& -\frac{A_{5}}{A_{1}+A_{2}+A_{3}} A_{5} f(2(x+y))-\frac{A_{6}+A_{7}}{A_{1}+A_{2}+A_{3}} A_{5} f(0) \\
& +\frac{A_{4}}{A_{1}+A_{2}+A_{3}} A_{5} f(-1(x+y)) \\
& -\frac{A_{5}}{A_{1}+A_{2}+A_{3}} A_{6} f(2(x+z))-\frac{A_{6}+A_{7}}{A_{1}+A_{2}+A_{3}} A_{6} f(0) \\
& +\frac{A_{4}}{A_{1}+A_{2}+A_{3}} A_{5} f(-1(x+z)) \\
& -\frac{A_{5}}{A_{1}+A_{2}+A_{3}} A_{7} f(2(y+z))-\frac{A_{6}+A_{7}}{A_{1}+A_{2}+A_{3}} A_{7} f(0) \\
& +\frac{A_{4}}{A_{1}+A_{2}+A_{3}} A_{7} f(-1(y+z)) \| \\
& \leq\left|\frac{A_{5}}{A_{1}+A_{2}+A_{3}}\right| L(2 x, 2 y, 2 z)+\left|\frac{A_{6}+A_{7}}{A_{1}+A_{2}+A_{3}}\right| L(0,0,0) \\
& +\left|\frac{A_{4}}{A_{1}+A_{2}+A_{3}}\right| L(-x,-y,-z) \\
& \leq(d(2) c(2)+d(0) c(0)+d(-1) c(-1)) L(x, y, z) \\
& =b L(x, y, z) \text {. }
\end{aligned}
$$

Now, suppose that (29) holds for some $n \in \mathbb{N}_{0}$. Then, for every $(x, y, z) \in X^{3}$, we have: 


$$
\begin{aligned}
& \| A_{1} \mathcal{T}^{n+1} f(x+y+z)+A_{2} \mathcal{T}^{n+1} f(x)+A_{3} \mathcal{T}^{n+1} f(y)+A_{4} \mathcal{T}^{n+1} f(z) \\
& A_{5} \mathcal{T}^{n+1} f(x+y)-A_{6} \mathcal{T}^{n+1} f(x+z)-A_{7} \mathcal{T}^{n+1} f(y+z) \| \\
& \left.=\| \frac{A_{5}}{A_{1}+A_{2}+A_{3}} A_{1} \mathcal{T}^{n} f(2(x+y+z))+\frac{A_{6}+A_{7}}{A_{1}+A_{2}+A_{3}} A_{1} \mathcal{T}^{n} f(0)\right) \\
& -\frac{A_{4}}{A_{1}+A_{2}+A_{3}} A_{1} \mathcal{T}^{n} f(-(x+y+z)) \\
& +\frac{A_{5}}{A_{1}+A_{2}+A_{3}} A_{2} \mathcal{T}^{n} f(2 x)+\frac{A_{6}+A_{7}}{A_{1}+A_{2}+A_{3}} A_{2} \mathcal{T}^{n} f(0) \\
& -\frac{A_{3}}{A_{1}+A_{2}+A_{3}} A_{2} \mathcal{T}^{n} f(-x) \\
& +\frac{A_{5}}{A_{1}+A_{2}+A_{3}} A_{3} \mathcal{T}^{n} f(2 y)+\frac{A_{6}+A_{7}}{A_{1}+A_{2}+A_{3}} A_{3} \mathcal{T}^{n} f(0) \\
& -\frac{A_{4}}{A_{1}+A_{2}+A_{3}} A_{3} \mathcal{T}^{n} f(-y) \\
& +\frac{A_{5}}{A_{1}+A_{2}+A_{3}} A_{4} \mathcal{T}^{n} f(2 z)+\frac{A_{6}+A_{7}}{A_{1}+A_{2}+A_{3}} A_{4} \mathcal{T}^{n} f(0) \\
& -\frac{A_{4}}{A_{1}+A_{2}+A_{3}} A_{4} \mathcal{T}^{n} f(-z) \\
& -\frac{A_{5}}{A_{1}+A_{2}+A_{3}} A_{5} \mathcal{T}^{n} f(2(x+y))-\frac{A_{6}+A_{7}}{A_{1}+A_{2}+A_{3}} A_{5} \mathcal{T}^{n} f(0) \\
& +\frac{A_{4}}{A_{1}+A_{2}+A_{3}} A_{5} \mathcal{T}^{n} f(-1(x+y)) \\
& -\frac{A_{5}}{A_{1}+A_{2}+A_{3}} A_{6} \mathcal{T}^{n} f(2(x+z))-\frac{A_{6}+A_{7}}{A_{1}+A_{2}+A_{3}} A_{6} \mathcal{T}^{n} f(0) \\
& +\frac{A_{4}}{A_{1}+A_{2}+A_{3}} A_{5} \mathcal{T}^{n} f(-1(x+z)) \\
& -\frac{A_{5}}{A_{1}+A_{2}+A_{3}} A_{7} \mathcal{T}^{n} f(2(y+z))-\frac{A_{6}+A_{7}}{A_{1}+A_{2}+A_{3}} A_{7} \mathcal{T}^{n} f(0) \\
& +\frac{A_{4}}{A_{1}+A_{2}+A_{3}} A_{7} \mathcal{T}^{n} f(-1(y+z)) \| \\
& \leq\left(\left|\frac{A_{5}}{A_{1}+A_{2}+A_{3}}\right| b^{n} L(2 x, 2 y, 2 z)+\left|\frac{A_{6}+A_{7}}{A_{1}+A_{2}+A_{3}}\right| b^{n} L(0,0,0)\right. \\
& \left.+\left|\frac{A_{4}}{A_{1}+A_{2}+A_{3}}\right| b^{n} L(-x,-y,-z)\right) \\
& \leq b^{n}(d(2) c(2)+d(0) c(0)+d(-1) c(-1)) L(x, y, z) \\
& =b^{n+1} L(x, y, z) \text {. }
\end{aligned}
$$

Thus, by induction, we obtain Condition (29). Letting $n \rightarrow \infty$ in (29), we get:

$$
\begin{aligned}
& A_{1} F(x+y+z)+A_{2} F(x)+A_{3} F(y)+A_{4} F(z) \\
& \quad=A_{5} F(x+y)+A_{6} F(x+z)+A_{7} F(y+z), \quad(x, y, z) \in X^{3} .
\end{aligned}
$$

Thus, we proved that there exists a function $F: X \rightarrow Y$ satisfying Equation (1) for all $x, y, z \in X$ and such that:

$$
\|f(x)-F(x)\| \leq \varepsilon^{*}(x) \leq \rho_{L}(x), \quad x \in X .
$$

Finally, we prove the uniqueness of the exact solution $F$ satisfying (19). To this end, we show by induction that for every $n \in \mathbb{N}$ :

$$
\left\|\mathcal{T}^{n} \xi(x)-\mathcal{T}^{n} \mu(x)\right\| \leq \Lambda^{n}(\|\xi-\mu\|)(x), \quad \xi, \mu \in Y^{X}, x \in X .
$$


For $n=1$, Condition (32) is simply (24). For $n=2$ using (24) and the monotonicity of $\Lambda$, we have:

$$
\begin{aligned}
\left\|\mathcal{T}^{2} \xi(x)-\mathcal{T}^{2} \mu(x)\right\| & =\|\mathcal{T}(\mathcal{T} \xi)(x)-\mathcal{T}(\mathcal{T} \mu)(x)\| \leq \Lambda(\|\mathcal{T} \xi-\mathcal{T} \mu\|)(x) \\
& \leq \Lambda(\Lambda(\|\xi-\mu\|))(x)=\Lambda^{2}(\|\xi-\mu\|)(x), \quad x \in X
\end{aligned}
$$

for $\xi, \mu \in Y^{X}$. Fix $\xi, \mu \in Y^{X}$, and assume that for an $n \in \mathbb{N}$, Relation (32) holds. Then, by (24):

$$
\begin{array}{r}
\left\|\mathcal{T}^{n+1} \xi(x)-\mathcal{T}^{n+1} \mu(x)\right\|=\left\|\mathcal{T}\left(\mathcal{T}^{n} \xi\right)(x)-\mathcal{T}\left(\mathcal{T}^{n} \mu\right)(x)\right\| \\
\leq \Lambda\left(\left\|\mathcal{T}^{n} \xi-\mathcal{T}^{n} \mu\right\|\right)(x), \quad x \in X .
\end{array}
$$

Hence, by the inductive hypothesis and the monotonicity of $\Lambda$, we obtain:

$$
\left\|\mathcal{T}^{n+1} \xi(x)-\mathcal{T}^{n+1} \mu(x)\right\| \leq \Lambda\left(\Lambda^{n}(\|\xi-\mu\|)\right)(x)=\Lambda^{n+1}(\|\xi-\mu\|)(x), \quad x \in X .
$$

Let $G: X \rightarrow Y$ be also a solution of (1) such that $\|f(x)-G(x)\| \leq \rho_{L}(x)$ for $x \in X$. Then:

$$
\|G(x)-F(x)\| \leq 2 \rho_{L}(x), \quad x \in X
$$

Hence, by (32), we obtain:

$$
\left\|\mathcal{T}^{n} G(x)-\mathcal{T}^{n} F(x)\right\| \leq 2 \Lambda^{n} \rho_{L}(x) \leq \frac{2 \Lambda^{n} \varepsilon(x)}{1-\gamma(x)}, \quad x \in X,
$$

since $\Lambda$ is linear and monotone. Letting $n \rightarrow \infty$, by the convergence of the series $\sum_{n=0}^{\infty} \Lambda^{n} \varepsilon(x)$, we get:

$$
\lim _{n \rightarrow \infty}\left\|\mathcal{T}^{n} G(x)-\mathcal{T}^{n} F(x)\right\|=0, \quad x \in X
$$

Hence, $\|G(x)-F(x)\|=0$ for $x \in X$, since $G$ and $F$ are fixed points of $\mathcal{T}$. Consequently, $G(x)=F(x)$ for every $x \in X$.

Let us point out that the numerator $\left|A_{6}+A_{7}\right|$ of the constant $d(0)$ can be much smaller than the sum $\left|A_{6}\right|+\left|A_{7}\right|$ in the case where the numbers $A_{6}, A_{7}$ have opposite signs. Therefore, for some values of coefficients $A_{i}$, the above theorem can give the better approximation of the exact solution of Equation (1) than the known results. Moreover, replacing $\left|A_{6}\right|+\left|A_{7}\right|$ with $\left|A_{6}+A_{7}\right|$ causes a larger set of coefficients to be covered by the stability results, since the above theorem contains the assumption $b<1$, and analogous assumptions occur in the known results concerning the stability of more general equations.

Let us note that if $L(0,0,0) \neq 0$, then by (16), we get that $c(0) \geq 1$. However, in the case where $L(0,0,0)=0$, without loss of generality, we can put $c(0)=0$. Then, the constant $b$ occurring in (20) is of the form:

$$
b=c(2) d(2)+c(-1) d(-1)
$$

where $d(2)$ and $d(-1)$ are given by (17). Hence, if $A_{4}=A_{5}=0$, then $d(2)=d(-1)=0$. Thus, $b=0$, and consequently, $\varepsilon^{*}(x)=\varepsilon(x)$. In this case, we can easily determine the exact solution of the considered functional equation.

Corollary 1. Let $(X,+)$ be an abelian group, $Y$ be a Banach space, and $A_{1}, \ldots, A_{7} \in \mathbb{K} \in\{\mathbb{R}, \mathbb{C}\}$. Assume that $A_{1}+A_{2}+A_{3} \neq 0$ and $A_{4}=A_{5}=0$. Let a function $L: X^{3} \rightarrow[0, \infty)$ satisfy the conditions $L(0,0,0)=0$ and: 


$$
L(k x, k y, k z) \leq c(k) L(x, y, z), \quad(x, y, z) \in X^{3}, k \in\{2,-1\},
$$

with some $c(2), c(-1) \in[0, \infty)$. Assume that $f: X \rightarrow Y$ is a function such that Condition (18) is fulfilled. Then, there exists a unique function $F: X \rightarrow Y$ satisfying (1) such that:

$$
\|f(x)-F(x)\| \leq \rho_{L}(x), \quad x \in X,
$$

where:

$$
\rho_{L}(x):=\frac{L(x, x,-x)}{\left|A_{1}+A_{2}+A_{3}\right|}, \quad x \in X .
$$

Moreover, $F$ is a constant function given by the formula:

$$
F(x)=\frac{A_{6}+A_{7}}{A_{1}+A_{2}+A_{3}} f(0), \quad x \in X .
$$

Proof. From (22), under our assumption on coefficients $A_{i}$, we get that:

$$
\mathcal{T} \xi(t)=\frac{A_{6}+A_{7}}{A_{1}+A_{2}+A_{3}} \xi(0), \quad \xi \in Y^{X}, t \in X .
$$

Moreover, by (27), we have:

$$
F(t)=\frac{A_{6}+A_{7}}{A_{1}+A_{2}+A_{3}} F(0), \quad t \in X .
$$

By (17) and (34), we obtain that $b=0$. Hence, by (26):

$$
\Lambda^{n} \mathcal{E}(t)=0, \quad t \in X, n \in \mathbb{N},
$$

where:

$$
\varepsilon(t)=\frac{L(t, t,-t)}{\left|A_{1}+A_{2}+A_{3}\right|}, \quad t \in X .
$$

From (28), we obtain that $F(0)=f(0)$, since $L(0,0,0)=0$. Hence, by (36), we get Formula (35).

Now, we proceed to Case (V).

Theorem 3. Let $(X,+)$ be a commutative monoid, $Y$ be a Banach space, and $A_{1}, \ldots, A_{7} \in \mathbb{K} \in$ $\{\mathbb{R}, \mathbb{C}\}$. Assume that $-A_{2}-A_{3}+A_{6}+A_{7} \neq 0$. Let a function $L: X^{3} \rightarrow[0, \infty)$ satisfy the condition:

$$
L(k x, k y, k z) \leq c(k) L(x, y, z), \quad(x, y, z) \in X^{3}, k \in\{0,2\},
$$

with $c(2), c(0) \in[0, \infty)$ such that $b:=c(2) d(2)+c(0) d(0)<1$, where:

$$
d(2):=\left|\frac{A_{1}-A_{5}}{A_{6}+A_{7}-A_{2}-A_{3}}\right|, d(0):=\left|\frac{A_{4}}{A_{6}+A_{7}-A_{2}-A_{3}}\right| .
$$

Assume that $f: X \rightarrow Y$ is a function such that Condition (18) is fulfilled. Then, there exists a unique function $F: X \rightarrow Y$ satisfying (1) such that Condition (19) holds, where:

$$
\rho_{L}(x):=\frac{L(x, x, 0)}{\left|A_{6}+A_{7}-A_{2}-A_{3}\right|(1-b)}, \quad x \in X .
$$


Proof. Taking $x=t, y=t, z=0$ in (18), we obtain:

$$
\left\|\left(A_{6}+A_{7}-A_{2}-A_{3}\right) f(t)-\left(A_{1}-A_{5}\right) f(2 t)+A_{4} f(0)\right\| \leq L(t, t, 0), \quad t \in X .
$$

Hence, for each $x \in X$ :

$$
\left\|f(t)-\frac{A_{1}-A_{5}}{A_{6}+A_{7}-A_{2}-A_{3}} f(2 t)-\frac{A_{4}}{A_{6}+A_{7}-A_{2}-A_{3}} f(0)\right\| \leq \varepsilon(t),
$$

where $\varepsilon(t):=\frac{L(t, t, 0)}{\left|A_{6}+A_{7}-A_{2}-A_{3}\right|}$. Put:

$$
\mathcal{T} \xi(t):=\frac{A_{1}-A_{5}}{A_{6}+A_{7}-A_{2}-A_{3}} \xi(2 t)+\frac{A_{4}}{A_{6}+A_{7}-A_{2}-A_{3}} \xi(0), \quad \xi \in Y^{X}, t \in X .
$$

Let us note that the operator $\mathcal{T}$ is linear. From (40), we get that:

$$
\|f(t)-\mathcal{T} f(t)\| \leq \varepsilon(t), \quad t \in X
$$

Fix $\xi, \mu \in Y^{X}$. For every $t \in X$, we have:

$$
\begin{aligned}
\|\mathcal{T} \xi(t)-\mathcal{T} \mu(t)\|= & \left\|\frac{A_{1}-A_{5}}{A_{6}+A_{7}-A_{2}-A_{3}}(\xi(2 t)-\mu(2 t))+\frac{A_{4}}{A_{6}+A_{7}-A_{2}-A_{3}}(\xi(0)-\mu(0))\right\| \\
\leq & \left|\frac{A_{1}-A_{5}}{A_{6}+A_{7}-A_{2}-A_{3}}\right|\|\xi(2 t)-\mu(2 t)\| \\
& +\left|\frac{A_{4}}{A_{6}+A_{7}-A_{2}-A_{3}}\right|\|\xi(0)-\mu(0)\| .
\end{aligned}
$$

Thus:

$$
\|\mathcal{T} \xi(t)-\mathcal{T} \mu(t)\| \leq d(2)\|\xi(2 t)-\mu(2 t)\|+d(0)\|\xi(0)-\mu(0)\|, \quad t \in X
$$

We have shown that Condition (H2) is satisfied with $k=2, S=X, E=Y$,

$$
f_{1}(t)=2 t, \quad f_{2}(t)=0, \quad l_{1}(t)=d(2), \quad l_{2}(t)=d(0),
$$

i.e.,

$$
\|\mathcal{T} \xi(t)-\mathcal{T} \mu(t)\| \leq \sum_{i=1}^{2} l_{i}(t)\left\|\xi\left(f_{i}(t)\right)-\mu\left(f_{i}(t)\right)\right\|, \quad \xi, \mu \in Y^{X}, t \in X
$$

Define an operator $\Lambda: \mathbb{R}_{+}{ }^{X} \rightarrow \mathbb{R}_{+}{ }^{X}$ by:

$$
\Lambda \eta(t):=\sum_{i=1}^{2} l_{i}(t) \eta\left(f_{i}(t)\right), \quad t \in X
$$

for every $\eta \in \mathbb{R}_{+}{ }^{X}$. Then, for each $\eta \in \mathbb{R}_{+}{ }^{X}$, we have:

$$
\Lambda \eta(t):=d(2) \eta(2 t)+d(0) \eta(0), \quad t \in X
$$

Let us note that the operator $\Lambda$ is monotone, i.e., for all $\eta, \zeta \in \mathbb{R}_{+}{ }^{X}$ if $\eta \leq \zeta$, then $\Lambda \eta \leq \Lambda \zeta$. Moreover, by (42):

$$
\|\mathcal{T} \xi(t)-\mathcal{T} \mu(t)\| \leq \Lambda(\|\xi-\mu\|)(t), \quad \xi, \mu \in Y^{X}, t \in X .
$$


Now, we show that $\varepsilon^{*}(t):=\sum_{n=0}^{\infty} \Lambda^{n} \varepsilon(t)<\infty$ for each $t \in X$, i.e., the function series $\sum_{n=0}^{\infty} \Lambda^{n} \varepsilon(t)$ is convergent for each $t \in X$. Fix a $t \in X$. In view of (37), we have:

$$
\begin{aligned}
\Lambda \varepsilon(t) & =d(2) \varepsilon(2 t)+d(0) \varepsilon(0) \\
& =d(2) \frac{L(2 t, 2 t, 0)}{\left|A_{6}+A_{7}-A_{2}-A_{3}\right|}+d(0) \frac{L(0,0,0)}{\left|A_{6}+A_{7}-A_{2}-A_{3}\right|} \\
& \leq d(2) c(2) \frac{L(t, t, 0)}{\left|A_{6}+A_{7}-A_{2}-A_{3}\right|}+d(0) c(0) \frac{L(t, t, 0)}{\left|A_{6}+A_{7}-A_{2}-A_{3}\right|} \\
& =(d(2) c(2)+d(0) c(0)) \frac{L(t, t, 0)}{\left|A_{6}+A_{7}-A_{2}-A_{3}\right|} .
\end{aligned}
$$

Thus:

$$
\Lambda \varepsilon(t) \leq b \varepsilon(t), \quad t \in X
$$

By induction, we show that:

$$
\Lambda^{n} \varepsilon(t) \leq b^{n} \varepsilon(t), \quad t \in X
$$

For $n=1$, Condition (45) coincides with Condition (44). For $n=2$, by the monotonicity and linearity of $\Lambda$, we get from (44):

$$
\Lambda^{2} \varepsilon(t)=\Lambda(\Lambda \varepsilon)(t) \leq \Lambda(b \varepsilon)(t)=b \Lambda \varepsilon(t) \leq b^{2} \varepsilon(t)
$$

for all $t \in X$. Now, suppose that (45) holds for some $n \in \mathbb{N}$. Then, for every $t \in X$, using the inductive hypothesis, we have:

$$
\Lambda^{n+1} \mathcal{\varepsilon}(t)=\Lambda\left(\Lambda^{n} \mathcal{\varepsilon}\right)(t) \leq \Lambda\left(b^{n} \varepsilon\right)(t)=b^{n} \Lambda \mathcal{\varepsilon}(t) \leq b^{n+1} \mathcal{\varepsilon}(t) .
$$

Using (45), we obtain:

$$
\varepsilon^{*}(t)=\sum_{n=0}^{\infty} \Lambda^{n} \varepsilon(t) \leq \sum_{n=0}^{\infty} b^{n} \varepsilon(t)=\frac{\varepsilon(t)}{1-b}=\frac{L(t, t, 0)}{\left|A_{6}+A_{7}-A_{2}-A_{3}\right|(1-b)}
$$

for each $t \in X$. By Theorem 1 (with $S=X$ and $E=Y$ ), there exists a function $F: X \rightarrow Y$ such that:

$$
F(t)=\frac{A_{1}-A_{5}}{A_{6}+A_{7}-A_{2}-A_{3}} F(2 t)+\frac{A_{4}}{A_{6}+A_{7}-A_{2}-A_{3}} F(0), \quad t \in X,
$$

and:

$$
\|f(t)-F(t)\| \leq \varepsilon^{*}(t) \leq \frac{L(t, t, 0)}{\left|A_{6}+A_{7}-A_{2}-A_{3}\right|(1-b)}, \quad t \in X .
$$

Moreover,

$$
F(t)=\lim _{n \rightarrow \infty} \mathcal{T}^{n} f(t), \quad t \in X .
$$

Next, by induction, we show that for every $(x, y, z) \in X^{3}, n \in \mathbb{N}_{0}:=\mathbb{N} \cup\{0\}$ :

$$
\begin{aligned}
\| A_{1} \mathcal{T}^{n} f(x+y+z) & +A_{2} \mathcal{T}^{n} f(x)+A_{3} \mathcal{T}^{n} f(y)+A_{4} \mathcal{T}^{n} f(z) \\
& -A_{5} \mathcal{T}^{n} f(x+y)-A_{6} \mathcal{T}^{n} f(x+z)-A_{7} \mathcal{T}^{n} f(y+z) \| \\
\leq & b^{n} L(x, y, z),(x, y, z) \in X^{3} .
\end{aligned}
$$

For $n=0$, Condition (46) is simply (18). For $n=1$ using (41), we have: 


$$
\begin{aligned}
\| A_{1} \mathcal{T} f( & +y+z)+A_{2} \mathcal{T} f(x)+A_{3} \mathcal{T} f(y)+A_{4} \mathcal{T} f(z) \\
& -A_{5} \mathcal{T} f(x+y)-A_{6} \mathcal{T} f(x+z)-A_{7} \mathcal{T} f(y+z) \| \\
= & \| \frac{A_{1}-A_{5}}{A_{6}+A_{7}-A_{2}-A_{3}} A_{1} f(2(x+y+z))+\frac{A_{4}}{A_{6}+A_{7}-A_{2}-A_{3}} A_{1} f(0) \\
& +\frac{A_{1}-A_{5}}{A_{6}+A_{7}-A_{2}-A_{3}} A_{2} f(2 x)+\frac{A_{4}}{A_{6}+A_{7}-A_{2}-A_{3}} A_{2} f(0) \\
& +\frac{A_{1}-A_{5}}{A_{6}+A_{7}-A_{2}-A_{3}} A_{3} f(2 y)+\frac{A_{4}}{A_{6}+A_{7}-A_{2}-A_{3}} A_{3} f(0) \\
& +\frac{A_{1}-A_{5}}{A_{6}+A_{7}-A_{2}-A_{3}} A_{4} f(2 z)+\frac{A_{4}}{A_{6}+A_{7}-A_{2}-A_{3}} A_{4} f(0) \\
& -\frac{A_{1}-A_{5}}{A_{6}+A_{7}-A_{2}-A_{3}} A_{5} f(2(x+y))-\frac{A_{4}}{A_{6}+A_{7}-A_{2}-A_{3}} A_{5} f(0) \\
& -\frac{A_{1}-A_{5}}{A_{6}+A_{7}-A_{2}-A_{3}} A_{6} f(2(x+z))-\frac{A_{4}}{A_{6}+A_{7}-A_{2}-A_{3}} A_{6} f(0) \\
& -\frac{A_{1}-A_{5}}{A_{6}+A_{7}-A_{2}-A_{3}} A_{7} f(2(y+z))-\frac{A_{4}}{A_{6}+A_{7}-A_{2}-A_{3}} A_{7} f(0) \\
\leq & \left|\frac{A_{1}-A_{5}}{A_{6}+A_{7}-A_{2}-A_{3}}\right| L(2 x, 2 y, 2 z)+\left|\frac{A_{4}}{A_{6}+A_{7}-A_{2}-A_{3}}\right| L(0,0,0) \\
\leq & (d(2) c(2)+d(0) c(0)) L(x, y, z) \\
= & b L(x, y, z) .
\end{aligned}
$$

Now, suppose that (46) holds for some $n \in \mathbb{N}_{0}$ and for every $(x, y, z) \in X^{3}$. Then, we have:

$$
\begin{array}{rl}
\| A_{1} \mathcal{T}^{n+1} & f(x+y+z)+A_{2} \mathcal{T}^{n+1} f(x)+A_{3} \mathcal{T}^{n+1} f(y)+A_{4} \mathcal{T}^{n+1} f(z) \\
& -A_{5} \mathcal{T}^{n+1} f(x+y)-A_{6} \mathcal{T}^{n+1} f(x+z)-A_{7} \mathcal{T}^{n+1} f(y+z) \| \\
= & \| \frac{A_{1}-A_{5}}{A_{6}+A_{7}-A_{2}-A_{3}} A_{1} \mathcal{T}^{n} f(2(x+y+z))+\frac{A_{4}}{A_{6}+A_{7}-A_{2}-A_{3}} A_{1} \mathcal{T}^{n} f(0) \\
& +\frac{A_{1}-A_{5}}{A_{6}+A_{7}-A_{2}-A_{3}} A_{2} \mathcal{T}^{n} f(2 x)+\frac{A_{4}}{A_{6}+A_{7}-A_{2}-A_{3}} A_{2} \mathcal{T}^{n} f(0) \\
& +\frac{A_{1}-A_{5}}{A_{6}+A_{7}-A_{2}-A_{3}} A_{3} \mathcal{T}^{n} f(2 y)+\frac{A_{4}}{A_{6}+A_{7}-A_{2}-A_{3}} A_{3} \mathcal{T}^{n} f(0) \\
& +\frac{A_{1}-A_{5}}{A_{6}+A_{7}-A_{2}-A_{3}} A_{4} \mathcal{T}^{n} f(2 z)+\frac{A_{4}}{A_{6}+A_{7}-A_{2}-A_{3}} A_{4} \mathcal{T}^{n} f(0) \\
& -\frac{A_{1}-A_{5}}{A_{6}+A_{7}-A_{2}-A_{3}} A_{5} \mathcal{T}^{n} f(2(x+y))-\frac{A_{4}}{A_{6}+A_{7}-A_{2}-A_{3}} A_{5} \mathcal{T}^{n} f(0) \\
& -\frac{A_{1}-A_{5}}{A_{6}+A_{7}-A_{2}-A_{3}} A_{6} \mathcal{T}^{n} f(2(x+z))-\frac{A_{4}}{A_{6}+A_{7}-A_{2}-A_{3}} A_{6} \mathcal{T}^{n} f(0) \\
& -\frac{A_{1}-A_{5}}{A_{6}+A_{7}-A_{2}-A_{3}} A_{7} \mathcal{T}^{n} f(2(y+z))-\frac{A_{4}}{A_{6}+A_{7}-A_{2}-A_{3}} A_{7} \mathcal{T}^{n} f(0) \\
\leq & \left|\frac{A_{1}-A_{5}}{A_{6}+A_{7}-A_{2}-A_{3}}\right| b^{n} L(2 x, 2 y, 2 z)+\left|\frac{A_{4}}{A_{6}+A_{7}-A_{2}-A_{3}}\right| b^{n} L(0,0,0) \\
\leq & (d(2) c(2)+d(0) c(0)) L(x, y, z) \\
= & b^{n+1} L(x, y, z) .
\end{array}
$$

Thus, by induction, we obtain Condition (46). Letting $n \rightarrow \infty$ in (46), we get:

$$
\begin{aligned}
& A_{1} F(x+y+z)+A_{2} F(x)+A_{3} F(y)+A_{4} F(z) \\
& \quad=A_{5} F(x+y)+A_{6} F(x+z)+A_{7} F(y+z), \quad(x, y, z) \in X^{3} .
\end{aligned}
$$


Therefore, we proved that there exists a function $F: X \rightarrow Y$ satisfying Equation (1) for all $x, y, z \in X$ and such that:

$$
\|f(x)-F(x)\| \leq \varepsilon^{*}(x) \leq \rho_{L}(x), \quad x \in X .
$$

Finally, we prove the uniqueness of the exact solution $F$ satisfying (19). To this end, we show by induction that for every $n \in \mathbb{N}$ :

$$
\left\|\mathcal{T}^{n} \xi(x)-\mathcal{T}^{n} \mu(x)\right\| \leq \Lambda^{n}(\|\xi-\mu\|)(x), \quad \xi, \mu \in Y^{X}, x \in X .
$$

For $n=1$, Condition (49) is simply (43). For $n=2$ using (43) and the monotonicity of $\Lambda$, we have:

$$
\begin{aligned}
\left\|\mathcal{T}^{2} \xi(x)-\mathcal{T}^{2} \mu(x)\right\| & =\|\mathcal{T}(\mathcal{T} \xi)(x)-\mathcal{T}(\mathcal{T} \mu)(x)\| \leq \Lambda(\|\mathcal{T} \xi-\mathcal{T} \mu\|)(x) \\
& \leq \Lambda(\Lambda(\|\xi-\mu\|))(x)=\Lambda^{2}(\|\xi-\mu\|)(x), \quad x \in X
\end{aligned}
$$

for $\xi, \mu \in Y^{X}$. Fix $\xi, \mu \in Y^{X}$, and assume that for an $n \in \mathbb{N}$, Relation (49) holds. Then, by (43):

$$
\begin{aligned}
\left\|\mathcal{T}^{n+1} \xi(x)-\mathcal{T}^{n+1} \mu(x)\right\| & =\left\|\mathcal{T}\left(\mathcal{T}^{n} \xi\right)(x)-\mathcal{T}\left(\mathcal{T}^{n} \mu\right)(x)\right\| \\
\leq & \Lambda\left(\left\|\mathcal{T}^{n} \xi-\mathcal{T}^{n} \mu\right\|\right)(x), \quad x \in X
\end{aligned}
$$

Hence, by the inductive hypothesis and the monotonicity of $\Lambda$, we obtain:

$$
\left\|\mathcal{T}^{n+1} \xi(x)-\mathcal{T}^{n+1} \mu(x)\right\| \leq \Lambda\left(\Lambda^{n}(\|\xi-\mu\|)\right)(x)=\Lambda^{n+1}(\|\xi-\mu\|)(x), \quad x \in X .
$$

Let $G: X \rightarrow Y$ be also a solution of (1) such that $\|f(x)-G(x)\| \leq \rho_{L}(x)$ for $x \in X$. Then:

$$
\|G(x)-F(x)\| \leq 2 \rho_{L}(x), \quad x \in X .
$$

Hence, by (49), we obtain:

$$
\left\|\mathcal{T}^{n} G(x)-\mathcal{T}^{n} F(x)\right\| \leq 2 \Lambda^{n} \rho_{L}(x) \leq \frac{2 \Lambda^{n} \varepsilon(x)}{1-\gamma(x)}, \quad x \in X,
$$

since $\Lambda$ is linear and monotone. Letting $n \rightarrow \infty$, by the convergence of the series $\sum_{n=0}^{\infty} \Lambda^{n} \mathcal{E}(x)$, we get:

$$
\lim _{n \rightarrow \infty}\left\|\mathcal{T}^{n} G(x)-\mathcal{T}^{n} F(x)\right\|=0, \quad x \in X .
$$

Hence, $\|G(x)-F(x)\|=0$ for $x \in X$, since $G$ and $F$ are fixed points of $\mathcal{T}$. Consequently, $G(x)=F(x)$ for every $x \in X$.

Similar as before, let us note that if $L(0,0,0) \neq 0$, then by (16), we get that $c(0) \geq 1$. However, in the case where $L(0,0,0)=0$, without loss of generality, we can put $c(0)=0$. Then, the constant $b$ occurring in (39) is of the form:

$$
b=c(2) d(2),
$$

where $d(2)$ is given by (38). If $A_{1}=A_{5}$, then $b=0$, and consequently, $\varepsilon^{*}(x)=\varepsilon(x)$.

Corollary 2. Let $(X,+)$ be a commutative monoid, $Y$ be a Banach space, and $A_{1}, \ldots, A_{7} \in \mathbb{K} \in$ $\{\mathbb{R}, \mathbb{C}\}$. Assume that $A_{6}+A_{7}-A_{2}-A_{3} \neq 0$ and $A_{1}=A_{5}$. Let a function $L: X^{3} \rightarrow[0, \infty)$ satisfy the conditions $L(0,0,0)=0$ and:

$$
L(k x, k y, k z) \leq c(2) L(x, y, z), \quad(x, y, z) \in X^{3},
$$


with a constant $c(2) \in[0, \infty)$. Assume that $f: X \rightarrow Y$ is a function such that Condition (18) is fulfilled. Then, there exists a unique function $F: X \rightarrow Y$ satisfying (1) such that Condition (19) holds, where:

$$
\rho_{L}(x):=\frac{L(x, x, 0)}{\left|A_{6}+A_{7}-A_{2}-A_{3}\right|}, \quad x \in X .
$$

Moreover, $F$ is a constant function given by the formula:

$$
F(x)=\frac{A_{4}}{A_{6}+A_{7}-A_{2}-A_{3}} f(0), \quad x \in X .
$$

\section{Applications}

For each of Cases (I)-(VII), we can obtain the estimation:

$$
\|f(x)-F(x)\| \leq \rho_{L}(x)
$$

of the distance between an approximate solution of Equation (1) and its exact solution obtained by using Theorem 1 . We list below the formula for the function $\rho_{L}$ and respective constants for consecutive cases (cf. Relations (4)-(10)).

(I) $\quad \rho_{L}(x):=\frac{L(x, x, x)}{\left|A_{6}+A_{7}-A_{2}-A_{3}\right|(1-b)}, \quad b:=c(2) d(2)+c(3) d(3)<1$,

$$
d(2):=\left|\frac{A_{5}+A_{6}+A_{7}}{A_{2}+A_{3}+A_{4}}\right|, d(3):=\left|\frac{A_{1}}{A_{2}+A_{3}+A_{4}}\right| ;
$$

(II) $\left.\quad \rho_{L}(x):=\frac{L(x, x,-x)}{\left|A_{1}+A_{2}+A_{3}\right|(1-b)}, \quad b:=c(2) d(2)+c(0) d(0)+c(-1) d(-1)\right)<1$,

$$
d(2):=\left|\frac{A_{5}}{A_{1}+A_{2}+A_{3}}\right|, d(0):=\left|\frac{A_{6}+A_{7}}{A_{1}+A_{2}+A_{3}}\right|, d(-1):=\left|\frac{A_{4}}{A_{1}+A_{2}+A_{3}}\right| ;
$$

(III) $\left.\quad \rho_{L}(x):=\frac{L(x,-x, x)}{\left|A_{1}+A_{2}+A_{4}\right|(1-b)}, \quad b:=c(2) d(2)+c(0) d(0)+c(-1) d(-1)\right)<1$,

$$
d(2):=\left|\frac{A_{6}}{A_{1}+A_{2}+A_{4}}\right|, d(0):=\left|\frac{A_{5}+A_{7}}{A_{1}+A_{2}+A_{4}}\right|, d(-1):=\left|\frac{A_{3}}{A_{1}+A_{2}+A_{4}}\right| ;
$$

(IV) $\left.\rho_{L}(x):=\frac{L(-x, x, x)}{\left|A_{1}+A_{3}+A_{4}\right|(1-b)}, \quad b:=c(2) d(2)+c(0) d(0)+c(-1) d(-1)\right)<1$,

$$
d(2):=\left|\frac{A_{7}}{A_{1}+A_{3}+A_{4}}\right|, d(0):=\left|\frac{A_{5}+A_{6}}{A_{1}+A_{3}+A_{4}}\right|, d(-1):=\left|\frac{A_{2}}{A_{1}+A_{3}+A_{4}}\right| ;
$$

(V) $\left.\rho_{L}(x):=\frac{L(x, x, 0)}{\left|A_{6}+A_{7}-A_{2}-A_{3}\right|(1-b)}, \quad b:=c(2) d(2)+c(0) d(0)\right)<1$,

$$
d(2):=\left|\frac{A_{1}-A_{5}}{A_{6}+A_{7}-A_{2}-A_{3}}\right|, d(0):=\left|\frac{A_{4}}{A_{6}+A_{7}-A_{2}-A_{3}}\right|
$$

(VI) $\left.\quad \rho_{L}(x):=\frac{L(x, 0, x)}{\left|A_{5}+A_{7}-A_{2}-A_{4}\right|(1-b)}, \quad b:=c(2) d(2)+c(0) d(0)\right)<1$, 


$$
\begin{aligned}
& d(2):=\left|\frac{A_{1}-A_{6}}{A_{5}+A_{7}-A_{2}-A_{4}}\right|, d(0):=\left|\frac{A_{3}}{A_{5}+A_{7}-A_{2}-A_{4}}\right| ; \\
&\left.(\mathrm{VII}) \quad \rho_{L}(x):=\frac{L(0, x, x)}{\left|A_{5}+A_{6}-A_{3}-A_{4}\right|(1-b)}, \quad b:=c(2) d(2)+c(0) d(0)\right)<1, \\
& d(2):=\left|\frac{A_{1}-A_{7}}{A_{5}+A_{6}-A_{3}-A_{4}}\right|, d(0):=\left|\frac{A_{2}}{A_{5}+A_{6}-A_{3}-A_{4}}\right| .
\end{aligned}
$$

Now, we give some examples. Let $X$ be a normed space. Consider the control function $L: X^{3} \rightarrow[0, \infty)$ given by:

$$
L(x, y, z):=\|x\|^{p}+\|y\|^{p}+\|z\|^{p}, \quad(x, y, z) \in X^{3},
$$

with some $p \in \mathbb{R}$ such that $p>0$. It satisfies the condition:

$$
L(k x, k y, k z) \leq c(k) L(x, y, z), \quad(x, y, z) \in X^{3}
$$

with $c(k)=|k|^{p}$ for each $k \in \mathbb{Z}$, since $L(k x, k y, k z)=|k|^{p} L(x, y, z)$. Hence, $c(0)=0$ and $c(k) \geq 1$ for all $k \in \mathbb{Z} \backslash\{0\}$. Thus, for this $c$, we have the following assumptions:

(I) $\quad d(2)+d(3) \leq b<1, \quad d(2)+d(3)=\left|\frac{A_{5}+A_{6}+A_{7}}{A_{2}+A_{3}+A_{4}}\right|+\left|\frac{A_{1}}{A_{2}+A_{3}+A_{4}}\right| ;$

(II) $\quad d(2)+d(-1) \leq b<1, \quad d(2)+d(-1)=\left|\frac{A_{5}}{A_{1}+A_{2}+A_{3}}\right|+\left|\frac{A_{4}}{A_{1}+A_{2}+A_{3}}\right|$;

(III) $\quad d(2)+d(-1) \leq b<1, \quad d(2)+d(-1)=\left|\frac{A_{6}}{A_{1}+A_{2}+A_{4}}\right|+\left|\frac{A_{3}}{A_{1}+A_{2}+A_{4}}\right|$;

(IV) $\quad d(2)+d(-1) \leq b<1, \quad d(2)+d(-1)=\left|\frac{A_{7}}{A_{1}+A_{3}+A_{4}}\right|+\left|\frac{A_{2}}{A_{1}+A_{3}+A_{4}}\right|$;

(V) $\quad d(2) \leq b<1, \quad d(2)=\left|\frac{A_{1}-A_{5}}{A_{6}+A_{7}-A_{2}-A_{3}}\right|$;

(VI) $\quad d(2) \leq b<1, \quad d(2)=\left|\frac{A_{1}-A_{6}}{A_{5}+A_{7}-A_{2}-A_{4}}\right|$;

(VII) $\quad d(2) \leq b<1, \quad d(2)=\left|\frac{A_{1}-A_{7}}{A_{5}+A_{6}-A_{3}-A_{4}}\right|$.

Now, we show how one can use Theorems 2 and 3 to prove the stability of Equation (1) for some particular values of coefficients $A_{i}$. To apply Theorem 2, let us take:

$$
A_{1}=6, A_{2}=1, A_{3}=2, A_{4}=A_{5}=3, A_{6}=4, A_{7}=5 .
$$

By (17), we have:

$$
d(2)=\frac{1}{3}, d(-1)=\frac{1}{3}
$$


whence by (34), we get that:

$$
b=c(2) d(2)+c(-1) d(-1)=\frac{2^{p}}{3}+\frac{1}{3} .
$$

Consequently, $b<1$ if and only if $p \in(0,1)$. In particular, for $p=\frac{1}{2}$, we have $b=\frac{\sqrt{2}+1}{3}$ and:

$$
\rho_{L}(x)=\frac{L(x, x,-x)}{9\left(1-\frac{\sqrt{2}+1}{3}\right)}=\frac{2+\sqrt{2}}{2} \sqrt{\|x\|} .
$$

Thus:

$$
\|f(x)-F(x)\| \leq \frac{2+\sqrt{2}}{2} \sqrt{\|x\|,}
$$

where $F$ is of the form:

$$
F(x)=a(x)+c, \quad x \in X,
$$

with an additive function $a: X \rightarrow Y$ and $c=f(0)$.

Let us note that we obtain the same approximate if, e.g., $A_{6}=100$ and $A_{7}=-91$, since the constant $b$ occurring in Theorem 2 depends on $\left|A_{6}+A_{7}\right|$ and the values of $A_{6}$ and $A_{7}$ taken into account separately have no effect on $b$.

To prove the stability of Equation (1) for these values of the coefficients, we can also use Theorem 3. Then, by (38) and (51), we have $d(2)=\frac{1}{2}$ and:

$$
b=c(2) d(2)=\frac{2^{p}}{2} .
$$

Consequently, $b<1$ if and only if $p \in(0,1)$. In particular, for $p=\frac{1}{2}$, we have $b=\frac{\sqrt{2}}{2}$ and:

$$
\rho_{L}(x)=\frac{L(x, x, 0)}{6\left(1-\frac{\sqrt{2}}{2}\right)}=\frac{2+\sqrt{2}}{3} \sqrt{\|x\|} .
$$

Thus:

$$
\|f(x)-F(x)\| \leq \frac{2+\sqrt{2}}{3} \sqrt{\|x\|} .
$$

Summing up, for the considered values of coefficients, Theorem 3 gives a better approximation than Theorem 2, since in the case $p \in(0,1)$, we have:

$$
\frac{2^{p}}{2}<\frac{2^{p}}{3}+\frac{1}{3}
$$

Now, let us take:

$$
A_{1}=A_{2}=A_{3}=2, A_{4}=A_{5}=A_{6}=A_{7}=1 .
$$

First, we use Theorem 2. By (17), we have:

$$
d(2)=\frac{1}{6}, d(-1)=\frac{1}{6}
$$

whence by (34), we get that:

$$
b=c(2) d(2)+c(-1) d(-1)=\frac{2^{p}}{6}+\frac{1}{6} .
$$

Consequently, $b<1$ if and only if $p \in\left(0, \log _{2} 5\right)$. In particular, for $p=1$, we have $b=\frac{1}{2}$ and:

$$
\rho_{L}(x)=\frac{L(x, x,-x)}{6\left(1-\frac{1}{2}\right)}=\frac{L(x, x,-x)}{3}=\|x\| .
$$


For $p=\frac{1}{2}$, we have $b=\frac{\sqrt{2}+1}{6}$ and:

$$
\rho_{L}(x)=\frac{L(x, x,-x)}{6\left(1-\frac{\sqrt{2}+1}{6}\right)}=\frac{L(x, x,-x)}{5-\sqrt{2}}=\frac{3(5+\sqrt{2})}{23} \sqrt{\|x\|} \approx 0.8366 \sqrt{\|x\|} .
$$

To prove the stability of Equation (1) for these values of the coefficients, we can also use Theorem 3. Then, by (38) and (51), we have $d(2)=\frac{1}{2}$ and:

$$
b=c(2) d(2)=\frac{2^{p}}{2} \text {. }
$$

Consequently, $b<1$ if and only if $p \in(0,1)$. In particular, for $p=\frac{1}{2}$, we have $b=\frac{\sqrt{2}}{2}$ and:

$$
\rho_{L}(x)=\frac{L(x, x, 0)}{2\left(1-\frac{\sqrt{2}}{2}\right)}=(2+\sqrt{2}) \sqrt{\|x\|} \approx 3.4142 \sqrt{\|x\|} .
$$

Thus, now, Theorem 2 gives a better approximation than Theorem 3, since for $p>0$, we have:

$$
\frac{2^{p}}{6}+\frac{1}{6}<\frac{2^{p}}{2}
$$

Moreover, in this case, using Theorem 2, we obtain a wider interval for $p$.

\section{Final Remarks}

Let $L: X^{3} \rightarrow \mathbb{K}$ satisfy:

$$
L(k x, k y, k z) \leq c(k) L(x, y, z), \quad(x, y, z) \in X^{3}
$$

for some $k \in \mathbb{Z}$. Define $\widetilde{L}: X^{3} \rightarrow \mathbb{K}$ by the formula:

$$
\widetilde{L}(x, y, z):=L(x, y, z)+\delta,
$$

where $\delta>0$. Then, if $c(k) \geq 1$, then:

$$
\begin{aligned}
\widetilde{L}(k x, & k y, k z)=L(k x, k y, k z)+\delta \\
& \leq c(k) L(x, y, z)+\delta \\
& \leq c(k)\left(L(x, y, z)+\frac{\delta}{c(k)}\right) \\
& \leq c(k)(L(x, y, z)+\delta)=c(k) \widetilde{L}(x, y, z) .
\end{aligned}
$$

Let $L$ be given by (53). Then:

$$
\widetilde{L}(x, y, z)=\|x\|^{p}+\|y\|^{p}+\|z\|^{p}+\delta, \quad(x, y, z) \in X^{3} .
$$

and the condition:

$$
\widetilde{L}(k x, k y, k z) \leq \widetilde{c}(k) \widetilde{L}(x, y, z), \quad(x, y, z) \in X^{3}
$$

holds for each $k \in \mathbb{Z} \backslash\{0\}$ with $\widetilde{c}(k)=|k|^{p}$. For $k=0$, we have $\widetilde{L}(k x, k y, k z)=\delta$ for all $(x, y, z) \in X^{3}$. Hence, Relation (55) holds with $\widetilde{c}(0)=1$, since $\delta$ is the minimum of $L$. Thus, we obtain:

$$
\widetilde{c}(k):= \begin{cases}|k|^{p}, & \text { if } \quad k \neq 0, \\ 1, & \text { if } \quad k=0 .\end{cases}
$$

Let:

$$
A_{1}=A_{2}=A_{3}=2, A_{4}=A_{5}=A_{6}=A_{7}=1 .
$$


By Theorem 2

$$
\widetilde{b}=\widetilde{c}(2) d(2)+\widetilde{c}(0) d(0)+\widetilde{c}(-1) d(-1)=\frac{2^{p}+3}{6} .
$$

Hence, $\widetilde{b}<1$ if and only if $p \in\left(0, \log _{2} 3\right)$. For $p=1$, we have $\widetilde{b}=\frac{5}{6}$ and:

$$
\rho_{L}(x)=\frac{\widetilde{L}(x, x,-x)}{6\left(1-\frac{5}{6}\right)}=\widetilde{L}(x, x,-x)=3\|x\|^{p}+\delta .
$$

Now, let $A_{2}=A_{3}=2, A_{1}=A_{4}=A_{5}=A_{6}=A_{7}=1$. By Theorem 2, $\widetilde{b}=\frac{2^{p}+3}{5}$, since:

$$
d(2)=\frac{1}{5}, d(0)=\frac{2}{5}, d(-1)=\frac{1}{5}
$$

Then, $\widetilde{b}<1$ if and only if $p \in(0,1)$. In particular, for $p=\frac{1}{2}$, we obtain:

$$
b=\frac{3+\sqrt{2}}{5}, \rho_{L}(x)=\frac{\widetilde{L}(x, x,-x)}{5\left(1-\frac{3+\sqrt{2}}{5}\right)}=\frac{3 \sqrt{\|x\|}+\delta}{2-\sqrt{2}}=\frac{2+\sqrt{2}}{2}(3 \sqrt{\|x\|}+\delta) .
$$

By Theorem 3 with the same constants as above, we get that $\widetilde{b}=\frac{1}{2}$, and $\widetilde{b}<1$ if and only if $p \in(0, \infty)$, because $d(2)=0, d(0)=\frac{1}{2}$. The estimate is:

$$
\rho_{L}(x)=\frac{\widetilde{L}(x, x, 0)}{2\left(1-\frac{1}{2}\right)}=2\|x\|^{p}+\delta .
$$

For $p=\frac{1}{2}$, we have $\widetilde{b}=\frac{1}{2}$, and $\rho_{L}(x)=2 \sqrt{\|x\|}+\delta$.

\section{Discussion}

In order to obtain a solution of a generalized Fréchet functional equation, we used the iterative method based on a fixed point theorem. The distance between the obtained exact solution and the approximate solution being the starting point of the iterative process depends on the length of the first step of this process and the parameter $b$ controlling the length of the subsequent steps. The choice of the operator generating the iterative sequence tending to the exact solution has a big impact on the accuracy of the estimation of the distance between this solution and the initial approximate solution.

In this paper, we distinguished seven cases depending on the conditions met by the coefficients of the generalized Fréchet equation having constant coefficients. The result for the first of these cases was presented in [10]. It gives a good estimate for some values of the coefficients of the considered equation. However, for other values of the coefficients, these estimates may not be very accurate. Therefore, we distinguished other cases in order to compare the obtained estimates. For each of these cases, we provided an estimate of the distance between the exact and approximate solutions of the generalized Fréchet equation. For the given values of the coefficients of the equation, we can choose the case that gives a good estimate after checking if its assumptions are met. In particular, we showed an example of the coefficients for which Theorem 2 gives a better estimate than Theorem 3 and an example where Theorem 3 gives a more accurate result.

The system of linear equations defining the list of cases was selected so that, on the one hand, for any values of the coefficients, at least one of the cases was satisfied, and on the other hand, the operator defining the iterative sequence did not have too many summands. One can try to use such a system for a generalized Fréchet functional equation with variable coefficients, but then, it may happen that an equation of the system is satisfied for some values of $x, y$, and $z$, and for others, it does not hold. 


\section{Conclusions}

In this paper, we study the dependence of an estimation of the distance between approximate and exact solutions of the generalized Fréchet functional equation from the values of coefficients $A_{i}$ of this equation. We distinguish seven cases, of which at least one is always true. However, usually more than one of these cases holds, and then, we can choose the one that gives the best estimate among them. Generally speaking, we try to group the coefficients of the equation in a way that gives a good estimation of the distance between the approximate and exact solutions. The division into groups is important because within the groups, we sum up directly the coefficients and not their absolute values.

The desired estimate depends not only on the coefficients of the equation, but also on the control function $L$, as we showed in the examples. Nevertheless, grouping the coefficients may also be useful in investigating the stability of more general functional equations than the equation discussed in this article.

Author Contributions: Conceptualization, J.B.; methodology, J.B., Z.L., and R.M.; investigation, J.B., Z.L., and R.M.; writing, original draft preparation, R.M.; writing, review and editing, Z.L.; supervision, J.B. All authors read and agreed to the published version of the manuscript.

Funding: This research received no external funding.

Institutional Review Board Statement: Not applicable.

Informed Consent Statement: Not applicable.

Data Availability Statement: Not applicable.

Conflicts of Interest: The authors declare no conflict of interest.

\section{References}

1. Fréchet, M. Sur la définition axiomatique d'une classe d'espaces vectoriels distanciés applicables vectoriellement sur l'espace de Hilbert. Ann. Math. 1935, 36, 705-718. [CrossRef]

2. Alsina, C.; Sikorska, J.; Tomás, M.S. Norm Derivatives and Characterizations of Inner Product Spaces; World Scientific Publishing Co.: Singapore, 2010.

3. Bahyrycz, A.; Brzdęk, J.; Piszczek, M.; Sikorska, J. Hyperstability of the Fréchet equation and a characterization of inner product spaces. J. Funct. Spaces Appl. 2013, 2013, 496361. [CrossRef]

4. Dragomir, S.S. Some characterizations of inner product spaces and applications. Studia Univ. Babes-Bolyai Math. 1989, 34, 50-55.

5. Jordan, P.; von Neumann, J. On inner products in linear, metric spaces. Ann. Math. 1935, 36, 719-723. [CrossRef]

6. Moslehian, M.S.; Rassias, J.M. A Characterization of Inner Product Spaces Concerning an Euler-Lagrange Identity. Commun. Math. Anal. 2010, 8, 16-21.

7. Nikodem, K.; Páles, Z. Characterizations of inner product spaces by strongly convex functions. Banach J. Math. Anal. 2011, 5, 83-87. [CrossRef]

8. Rassias, T.M. New characterizations of inner product spaces. Bull. Sci. Math. 1984, 108, 95-99.

9. Bahyrycz, A.; Brzdęk, J.; Jabłońska, E.; Malejki, R. Ulam’s stability of a generalization of the Fréchet functional equation. J. Math. Anal. Appl. 2016, 442, 537-553. [CrossRef]

10. Brzdęk, J.; Leśniak, Z.; Malejki, R. On the generalized Fréchet functional equation with constant coefficients and its stability. Aequationes Math. 2018, 92, 355-373. [CrossRef]

11. Malejki, R. Stability of a generalization of the Fréchet functional equation. Ann. Univ. Paedagog. Crac. Stud. Math. 2015, 14, 69-79. [CrossRef]

12. Hyers, D.H.; Isac, G.; Rassias, T.M. Stability of Functional Equations in Several Variables; Birkhäuser: Boston, MA, USA, 1998.

13. Jung, S.-M. Hyers-Ulam-Rassias Stability of Functional Equations in Nonlinear Analysis; Springer Optimization and Its Applications; Springer: New York, NY, USA, 2011; Volume 48.

14. Kannappan, P. Functional Equations and Inequalities with Applications; Springer Monographs in Mathematics; Springer: New York, NY, USA, 2009.

15. Brillouët-Belluot, N.; Brzdęk, J.; Ciepliński, K. On some recent developments in Ulam's type stability. Abstr. Appl. Anal. 2012, 2012, 716936. [CrossRef]

16. Brzdęk, J.; Ciepliński, K.; Leśniak, Z. On Ulam’s type stability of the linear equation and related issues. Discret. Dyn. Nat. Soc. 2014, 2014, 536791. [CrossRef]

17. Jung, S.-M. On the Hyers-Ulam stability of the functional equation that have the quadratic property. J. Math. Anal. Appl. 1998, 222, 126-137. [CrossRef]

18. Brzdęk, J. Remarks on hyperstability of the Cauchy functional equation. Aequationes Math. 2013, 86, 255-267. [CrossRef] 
19. Brzdęk, J.; Jabłońska, E.; Moslehian, M.S.; Pacho, P. On stability of a functional equation of quadratic type. Acta Math. Hungar. 2016, 149, 160-169.

20. Fechner, W. On the Hyers-Ulam stability of functional equations connected with additive and quadratic mappings. J. Math. Anal. Appl. 2006, 322, 774-786. [CrossRef]

21. Gselmann, E. Hyperstability of a functional equation. Acta Math. Hungar. 2009, 124, 179-188. [CrossRef]

22. Lee, Y.-H. On the Hyers-Ulam-Rassias stability of the generalized polynomial function of degree 2. J. Chuncheong Math. Soc. 2009, 22, 201-209.

23. Maksa, G.; Páles, Z. Hyperstability of a class of linear functional equations. Acta Math. Acad. Paedag. Nyìregyháziensis 2001, 17, 107-112.

24. Phochai, T.; Saejung, S. Hyperstability of generalised linear functional equations in several variables. Bull. Aust. Math. Soc. 2020, 102, 293-302. [CrossRef]

25. Piszczek, M. Remark on hyperstability of the general linear equation. Aequationes Math. 2014, 88, 163-168. [CrossRef]

26. Popa, D.; Raşa, I. The Fréchet functional equation with application to the stability of certain operators. J. Approx. Theory 2012, 164, 138-144. [CrossRef]

27. Sikorska, J. On a direct method for proving the Hyers-Ulam stability of functional equations. J. Math. Anal. Appl. 2010, 372, 99-109. [CrossRef]

28. Brzdęk, J. Hyperstability of the Cauchy equation on restricted domains. Acta Math. Hung. 2013, 141, 58-67. [CrossRef]

29. Bahyrycz, A.; Olko, J. On stability of the general linear equation. Aequationes Math. 2015, 89, 1461-1474. [CrossRef]

30. Bahyrycz, A.; Olko, J. Hyperstability of general linear equation. Aequationes Math. 2016, 90, 527-540. [CrossRef]

31. Zhang, D. On Hyers-Ulam stability of generalized linear functional equation and its induced Hyers-Ulam programming problem. Aequat. Math. 2016, 90, 559-568. [CrossRef]

32. Zhang, D. On hyperstability of generalised linear functional equations in several variables. Bull. Aust. Math. Soc. 2015, 92, 259-267. [CrossRef]

33. Badora, R.; Brzdęk, J. Fixed points of a mapping and Hyers-Ulam stability. J. Math. Anal. Appl. 2014, 413, 450-457. [CrossRef]

34. Bahyrycz, A.; Brzdęk, J.; Leśniak, Z. On approximate solutions of the generalized Volterra integral equation. Nonlinear Anal. Real World Appl. 2014, 20, 59-66. [CrossRef]

35. Brzdęk, J.; Ciepliński, K. A fixed point approach to the stability of functional equations in non-Archimedean metric spaces. Nonlinear Anal. 2011, 74, 6861-6867. [CrossRef]

36. Cădariu, L.; Găvruţa, L.; Găvruţa, P. Fixed points and generalized Hyers-Ulam stability. Abstr. Appl. Anal. 2012, $2012,712743$. [CrossRef]

37. Brzdęk, J.; Chudziak, J.; Páles, Z. A fixed point approach to stability of functional equations. Nonlinear Anal. 2011, 74, 6728-6732. [CrossRef] 